\title{
On the Enhanced Heat Transfer in the Oscillatory Flow of Liquid Metals
}

\author{
K. Shailendhra ${ }^{1 \dagger}$ and S.P. AnjaliDevi ${ }^{2}$ \\ ${ }^{l}$ Department of Mathematics, Amrita School of Engineering, Amrita Vishwa Vidyapeetham, Ettimadai, Coimbatore, \\ Tamil Nadu 641 105, INDIA. \\ ${ }^{2}$ Department of Applied Mathematics, Bharathiar University, Coimbatore, Tamil Nadu, 641 046, INDIA.
}

†Corresponding Author Email: shailendhrak@gmail.com

(Received June 5, 2009; accepted February 24, 2010)

\begin{abstract}
The enhanced heat transfer in the oscillatory flow of liquid metals between two thermally insulated infinite parallel plates, when a constant axial temperature gradient superimposed, is investigated. The fluid is set to oscillation by oscillating both the plates axially along with an axial oscillatory body force, having the same frequency as that of the plates. The effective average thermal diffusivity is calculated and the effect of oscillation of the plates and the oscillatory body force on the enhancement of heat transfer are discussed and compared.
\end{abstract}

Keywords: Enhancement of heat transfer, Forced convection, Liquid metal flow.

\section{INTRODUCTION}

Modern engineering and technology is moving constantly upward on the temperature scale and subsequently the concept of enhancement of heat transfer has derived the attention of many scientists and engineers in the past few decades. Where formerly cold or softened metals was involved, liquid metal is now encountered; the steam engine and turbine have already given way to machines using liquid metal as the heat carrier and working medium; water-cooled atomic reactors are being replaced by reactors having a very high temperature zone cooled by actual streams of alkali metals, viz., sodium and potassium or their eutectic mixture.

A variety of methods have been considered so far to improve the heat transport capabilities of thermally conducting fluids. The interest in analytical studies and experimental designs of heat pipes started growing rapidly following the publication of the seminal paper by Grover et al. (1964). A very extensive amount of literature on the subject is currently available (Dunn and Reay 1978) and the heat pipe has been recognized as an important development in heat transfer technology. In spite of the various astounding applications of heat pipes, the rapid changes in modern technology quite often demand that scientists deal with very high temperatures and hence there is an everincreasing need to develop innovative methods for the enhancement of heat transfer.
Kurzweg and Zhao (1984) developed a novel heat transfer method in which heat is transported at very high rates from a hot to a cold fluid reservoir. In the novel heat transfer device, referred to as the "Dream Pipe" in the recent literature like Zhag et al. (2004), developed by Kurzweg (1986a), the basic idea is to transfer heat at high rates without concomitant net mass transfer by sinusoidal oscillation of liquid metals when a constant axial temperature gradient is superimposed on the fluid. Mathematically, the enhancement of heat transfer in this device due to sinusoidal oscillation of the fluid is based upon the combined effect of periodic longitudinal convective and lateral diffusive thermal energy transport in the presence of a longitudinal temperature gradient. Heat transfer rates in excess of those achievable with standard heat pipes are readily obtained by this thermal pumping process.

In their classical work Kurzweg and Zhao (1984) generated the oscillation of the fluid inside a bundle of capillary tubes by using external variable frequency shaker. It was established both experimentally and analytically that large quantities of heat is transported axially provided the fluid is oscillated at high frequency with large tidal displacement. It is also confirmed that under laminar conditions the radial variation in velocity and temperature produce an effective axial transport of heat, which is several orders of magnitude larger than that in the absence of oscillations. It was found that the effective averaged thermal diffusivity and the net heat 
flux per unit area in this novel method are very large and exceed those possible with the state of the art heatpipe technology by several orders of magnitude, provided the fluid is subjected to a constant axial thermal gradient. Indeed, Kurzweg (1985a), by using multiple time scale expansion technique showed that the heat flows as high as $10^{10} \mathrm{~W} / \mathrm{m}^{2}$ can be achieved in the oscillatory flow of fluids inside circular tubes.

The "Dream Pipe" designed by Kurzweg (1986a) can exhibit advantages, with respect to heat pipes, that the operating temperature can be selected optionally, only a low pressure needs to operate such pipes, the operation start and stop can be freely conducted, both wick device and the vacuum operation are not necessary, the structure is simple, and thereby such pipes can be easily manufactured. However, the "Dream Pipe" cannot be operated only by a temperature difference, the temperature difference though being sufficient to operate the conventional heat pipes. Therefore, an outside power for applying vibration to the liquid such as an electric motor or a compressor needs to be provided.

Kurzweg (1985b) analyzed the heat transfer enhancement in the oscillatory flow between thermally conducting parallel plates, when the fluid is set to oscillation by oscillatory pressure-gradient and subsequently investigated (Kurzweg 1986b) the temporal and spatial distribution of heat flux in the flow between two counter-oscillating thermally insulated plates. Kaviany (1986) analyzed the effects of viscous dissipation, channel spacing and presence of harmonics other than the fundamental harmonics on the enhancement of heat transfer. He concluded that the presence of frequencies lower than the fundamental could severely alter the performance. Ozawa and Kawamoto (1991) conducted numerical simulation and visualization experiments to investigate the fundamental heat transfer mechanism of this device.

Shailendhra and Anjali Devi (1997) analyzed the formation of a thermo-magnetic boundary layer and its role in the enhancement of heat transfer in MHD flow past an oscillating flat plate when the fluid is subjected to a constant longitudinal temperature gradient. Shailendhra (2002) carried out a detailed investigation on the hydrodynamic and hydromagnetic problems associated with this "dream pipe". Since liquid metals are employed in this novel device, Shailendhra and Anjali Devi (2005) also carried out an extensive study on the temporal and spatial distribution of heat flux in the enhancement of heat transfer in the MHD flow of liquid metals between counter oscillating thermally insulated parallel plates, when the fluid is subjected a constant axial temperature gradient.

Bergles (1985) provided a comprehensive description of the application and enhancement potential of heat exchangers. Effective heat exchange is critical to the process efficiency, and it significantly influences the economics of the design and the operation of the plant. Adverse effects increasing the cost and size of the heat exchangers can be eliminated (Manglik et al. 1992) with increased thermal performance of such equipments. Further, there is a great need from the industrial sector for different methods or techniques for augmenting the heat transfer in many areas so as to design reliable, economic and user-friendly devices that are specific to the required application. Only when such needs are met will the industrial and technological sectors give up the resistance to adopt new techniques of augmentation of heat transfer (Vishwas 1998).

It is quite natural to test whether there is a possibility to modify the design by changing the method of oscillation in the novel heat transfer mode proposed by Kurzweg (1984, 1985a) without spoiling the characteristic property of "enhanced heat transfer without concomitant net mass transfer under laminar conditions" so that this novel method may possibly find applications in many more areas where conventional heat pipes are now being used. It should be noted that in chemical, petrochemical, food, plastics, rubber and pharmaceutical plants, to name a few, heating and cooling of viscous process fluids are among the most important engineering problems and any study on this novel technique may find useful applications in all these fields.

So far, no attempt has been made to study explicitly and extensively the effect of changing the mode of oscillating the fluid over the enhancement of transfer, in this novel method, without changing the frequency from being fundamental. Thus, the purpose of the present analysis is to introduce two different modes of oscillating the fluid and to study their effects on the enhancement of heat transfer and also to compare the results with the earlier methods of oscillating the fluid. This present study may throw some light on the possibility of replacing heat pipes by suitable heat transfer devices based on this novel technique in many practical situations like thermal control of advanced communication spacecraft, cooling of semi-conductor power devices and solar energy collectors (Dunn and Reay 1978).

The hydrodynamics of enhanced longitudinal heat transfer in the oscillatory flow of liquid metals between two infinite, thermally insulated, horizontal, parallel plates, when a constant axial temperature gradient is superimposed on the fluid, is investigated. Here, the fluid is set to oscillation by oscillating both the plates axially along with an axial oscillatory body force, having the same frequency as that of the plates. The effective averaged thermal diffusivity is calculated and the effects of the various parameters on the enhancement of heat transfer are discussed.

\section{MATHEMATICAL ForMUlation}

Consider a Cartesian coordinate system centered between two parallel plates, where the direction of flow is taken as the $x-$ axis and the $y-$ axis is perpendicular to the two plates. The region $-h \leq y \leq h$ between the two plates is filled with viscous incompressible thermally conducting fluid such as liquid metals like liquid sodium, liquid potassium or their eutectic mixture. The two plates are oscillating in 
time with frequency $\omega$ in a direction parallel to themselves and an oscillatory body force $\vec{f}=\left(f_{x}, 0,0\right)$ , having the same frequency as that of the plates, is applied in the direction of motion of the plates. The fluid flow is characterized by the velocity field $\vec{q}=(u(y, t), 0,0)$. A constant temperature gradient $\gamma=\partial \theta / \partial x$ is superimposed on the fluid along the direction of the plates. The equation of continuity is automatically satisfied. The equation of motion is

$\frac{\partial u}{\partial t}=f_{x}+v \frac{\partial^{2} u}{\partial y^{2}}$

The physical quantities are cast in the suitable nondimensional form as described below: $Y=y / h, T=\omega t, F=f_{x} /\left(\omega^{2} \Delta x\right)$

and $U=u /(\omega \Delta x)$ so that Eq. (1) reduces to

$\frac{\partial U}{\partial T}=F+\frac{1}{\operatorname{Re}} \frac{\partial^{2} U}{\partial Y^{2}}$

where $Y, T, U$ and $F$ are non-dimensional space variable, time, velocity and body force per unit mass respectively. Here $\Delta x$ is the amplitude of oscillation of the plates and $\operatorname{Re}=\omega h^{2} / v$ is the Reynolds number.

Equation (2) is to be solved under the boundary conditions

$U=U_{O} \sin (T)$ at $\quad Y= \pm 1$

Here, $U_{o}$ is the maximum non-dimensional velocity of the plates.

The energy equation reduces to

$$
\frac{\partial \theta}{\partial T}+\Delta x U \gamma=\frac{1}{\beta^{2}} \frac{\partial^{2} \theta}{\partial Y^{2}}
$$

Here, $\theta$ is the temperature in Kelvin, $\beta=\left(h^{2} \omega / \kappa\right)^{1 / 2}$ is the frequency parameter, where $\kappa$ is the thermal diffusivity. Indeed, $\beta$ is a measure of magnitude of the thermal diffusion time to the oscillation period. In Eq. (4), the viscous dissipation term is neglected since the Prandtl numbers of liquid metals are very small. Further, it is to be noted that the axial temperature gradient $\partial \theta / \partial x$ is smaller in comparison to the much larger time-dependent transverse temperature gradient, which exists during most of the oscillation cycles. This means $\partial^{2} \theta / \partial y^{2}$ is much larger than $\partial^{2} \theta / \partial x^{2}$, while the value of $\partial \theta / \partial x$ is taken to be equal to the time-averaged value $\gamma$ (Kurzweg 1986b).

The corresponding boundary conditions in the $Y$ direction appropriate to the geometry, the plates being insulated, are $\frac{\partial \theta}{\partial Y}=0 \quad$ at $\quad Y= \pm 1$

\section{Solution Of The Problem}

Equation (2) subjected to boundary condition (3) can be solved by the method of separation of variables. Assuming

$$
F=F_{O} \operatorname{Im}\{\exp (i T)\} \text { and } U=\operatorname{Im}\{\Phi(Y) \exp (i T)\}
$$

where $\mathrm{Im}$ denotes the imaginary part of the complex quantities and $F_{O}$ denotes the maximum nondimensional body force per unit mass, it is easy to see that

$$
\Phi(Y)=\left[\frac{(E(Y)+i G(Y))\left(U_{O}+i F_{O}\right)}{E+i G}\right]-i F_{O}
$$

where

$$
\begin{aligned}
& E=\cosh (\vartheta) \cos (\vartheta) \\
& G=\sinh (\vartheta) \sin (\vartheta) \\
& E(Y)=\cosh (\vartheta Y) \cos (\vartheta Y) \\
& G(Y)=\sinh (\vartheta Y) \sin (\vartheta Y)
\end{aligned}
$$

with $\vartheta=\sqrt{(\operatorname{Re} / 2)}$.

To solve for $\theta$, a temperature distribution of the following form is assumed:

$$
\theta(x, Y, t)=\gamma\{x+h \operatorname{Im}[g(Y) \exp (i \omega t)]\}
$$

Here, Im denotes the imaginary part of the complex quantity within the brackets. This form was first proposed by Chatwin (1975), in his problem on the longitudinal dispersion of passive contaminant in oscillatory flows in tubes. Owing to the mathematical similarity of the equation of dispersion of contaminants and the heat equation, it was adopted by Kurzweg (1985 b, 1986 b).

From Eqs. (4), (5) and (7), it is evident that

$\frac{d^{2} g}{d Y^{2}}-i \beta^{2} g=\operatorname{Pe} \Phi(Y)$

$g^{\prime}(Y)=0 \quad$ at $\quad Y= \pm 1$

Here, $P e=(\omega \Delta x) h / \kappa$ is the Peclect number. Solving Eq. (8) and using Eq. (9), it follows that

$$
g(Y)=A \cosh (\sqrt{i} \beta Y)+g_{1}(Y)
$$

Here, $A=A_{1}\left(A_{2}+i A_{3}\right)$ where

$$
\begin{aligned}
& A_{1}=-\frac{P e \sqrt{\mathrm{Re}}}{2 \beta\left(\beta^{2}-\mathrm{Re}\right)\left(E^{2}+G^{2}\right)\left(H_{1}^{2}+K_{1}^{2}\right)} \\
& A_{2}=\left(H_{1}-K_{1}\right)\left(B_{1}-C_{1}\right)+\left(H_{1}+K_{1}\right)\left(B_{2}+C_{2}\right) \\
& A_{3}=\left(H_{1}-K_{1}\right)\left(B_{3}+C_{2}\right)-\left(H_{1}+K_{1}\right)\left(B_{4}-C_{1}\right) \\
& B_{1}=U_{o}[(H-K) G-(H+K) E] \\
& B_{2}=U_{o}[(H-K) E+(H+K) G] \\
& B_{3}=U_{o}[(H-K) E-(H+K) G]
\end{aligned}
$$




$$
\begin{aligned}
& B_{4}=U_{O}[(H-K) G+(H+K) E] \\
& C_{1}=F_{O}(H+K) G+(H-K) E \\
& C_{2}=F_{O}(H-K) G-(H+K) E \\
& H=\sinh (\vartheta) \cos (\vartheta) \\
& K=\cosh (\vartheta) \sin (\vartheta) \\
& H_{1}=\sinh (\beta / \sqrt{2}) \cos (\beta / \sqrt{2}) \\
& K_{1}=\cosh (\beta / \sqrt{2}) \sin (\beta / \sqrt{2}) \\
& g_{1}(Y)=D_{1}\left\{D_{2} \varphi_{1}(Y)+D_{3} \varphi_{2}(Y)\right\}+D_{4} \\
& D_{1}=P e /\left(E^{2}+G^{2}\right) ; D_{2}=U_{O} E+F_{O} G \\
& D_{3}=U_{O} G-F_{O} E ; D_{4}=P e F_{O} / \beta^{2} \\
& \varphi_{1}(Y)=f_{1}(Y)+i f_{2}(Y) \\
& \varphi_{2}(Y)=f_{2}(Y)-i f_{1}(Y) \\
& f_{1}(Y)=D_{5}\left[-\operatorname{Re} G(Y)+i \beta^{2} E(Y)\right] \\
& f_{2}(Y)=D_{5}\left[\operatorname{Re} E(Y)+i \beta^{2} G(Y)\right] \\
& D_{5}=1 /\left(\beta^{4}-\operatorname{Re}^{2}\right)
\end{aligned}
$$

\section{ENHANCED ThERMAL DifFusivity}

The enhanced heat transfer that can be expected due to the oscillation of the fluid may be evaluated. The effective averaged thermal diffusivity $\kappa_{e}$ can be defined by the equality (Kurzweg 1985b)

$$
\kappa_{e} \gamma=\kappa \gamma-(1 / 2 h) \int_{-h}^{h}\left\{(\omega / 2 \pi) \int_{0}^{2 \pi / \omega} \theta u d t\right\} d y
$$

The left-hand side of Eq. (11) represents the effective axial thermal flux per unit cross sectional area and the right-hand side characterizes the time-averaged convective thermal flux produced by the interaction of the cross-stream varying velocity and temperature. It is to be noticed that the quantities in this integrand do not average out to zero over time so that there will be a net flow, whereas obviously, the time average of the velocity will be zero so that there can be no net accompanying mass transfer.

Finally, we define the non-dimensional effective averaged thermal diffusivity as

$$
\dot{\lambda}=\left(\kappa_{e}-\kappa\right) /\left(\omega \Delta x^{2}\right) \text {. }
$$

It is easy to see that

$\dot{\lambda}=\frac{-\beta^{2}}{4} \int_{-1}^{+1}\left(\Phi_{R} g_{1 R}+\Phi_{I} g_{1 I}\right) d Y$

where $g_{1 R}=g_{R} / P e$ and $g_{1 I}=g_{I} / P e$. Here, the suffix $R$ and $I$ denote the real and imaginary parts of the respective functions.

\section{RESULTS AND DISCUSSION}

It is to be noted that $U_{O}$ and $F_{O}$ represent the maximum non-dimensional velocity of the plates and body force per unit mass respectively and as the fluid motion is generated by both the oscillations of the plates and the oscillatory body force, it is evident that the velocity of the fluid can be controlled by $F_{O}$ and $U_{O}$ and as a consequence the effective averaged thermal diffusivity $\dot{\lambda}$ depends on these two controlling parameters. Therefore, further discussion is focused on the effect of these parameters on $\dot{\lambda}$.

In general, it is noted that the present methods of oscillating the fluid, either by oscillatory body force or by oscillations of the plates or both, maintain the characteristic feature of the dream pipe designed by Kurzweg (1986a).

From Figs. 1 to 5, it is evident that whatever may be the value of $U_{O}$ and $F_{O}, \dot{\lambda}$ attains its maximum value whenever $\beta=\pi$. It is observed that the maximum value of $\dot{\lambda}$ is always $10^{4}$ times greater than the actual thermal diffusivity without the oscillation of the fluid (when $\beta=0$ ). Thus, due to oscillation of the fluid, there is an increase in the effective thermal diffusivity $\kappa_{e}$ by several orders of magnitude is comparison to the actual thermal diffusivity $\kappa$ and such an increase is proportional to the frequency $\omega$ and square of the amplitude of oscillation of the plates $\Delta x$. However, one cannot indefinitely increase the values of the $\omega$ and $\Delta x^{2}$ since laminar conditions are to be maintained in order to ensure zero net mass transfer. Fortunately, for the optimum value of $\beta=\pi$, laminar conditions can be maintained (Kurzweg 1985b; Merkli and Thomann 1975).



Fig. 1. Effect of oscillatory body force on $\dot{\lambda}$ in the absence of oscillation of plates

It is clear from Fig. 1 that when the plates are at rest, the maximum value of $\dot{\lambda}$ increases by a factor 10 approximately if the amplitude of the body force is increased from one to three. Again, there is a substantial increase in the maximum value of $\dot{\lambda}$ due to a small increase in $F_{O}$ when the plates are set to oscillation, which is depicted in Fig. 2. The profiles of Figs. 3 and 4 are very similar to those of Figs. 1 and 2 respectively. Thus, it is inferred that the effect of oscillation of the 
plates on $\dot{\lambda}$, in the absence of body force, is the same as the effect of body force on it, in the absence of oscillation of the plates. Similarly, the effect of oscillation of the plates on $\dot{\lambda}$, in the presence of body force, is the same as the effect of body force on it, when the plates are set to oscillation. This result can be of much use while designing heat transfer devices based on this novel heat transfer mode to meet specific requirements of the area of application.

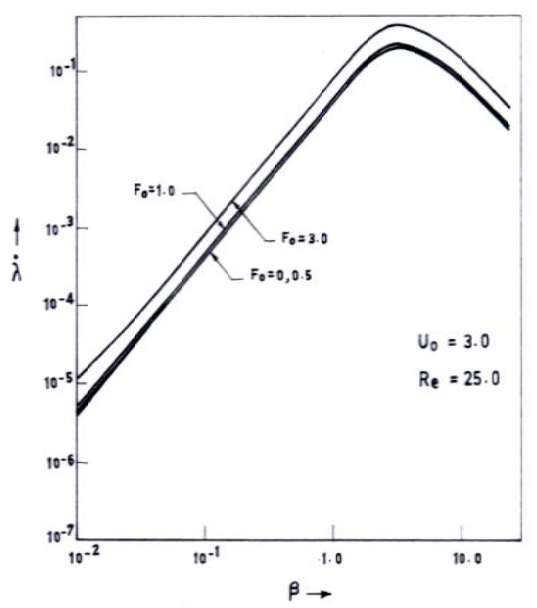

Fig. 2. Effect of oscillatory body force on $\dot{\lambda}$ in the presence of oscillation of plates

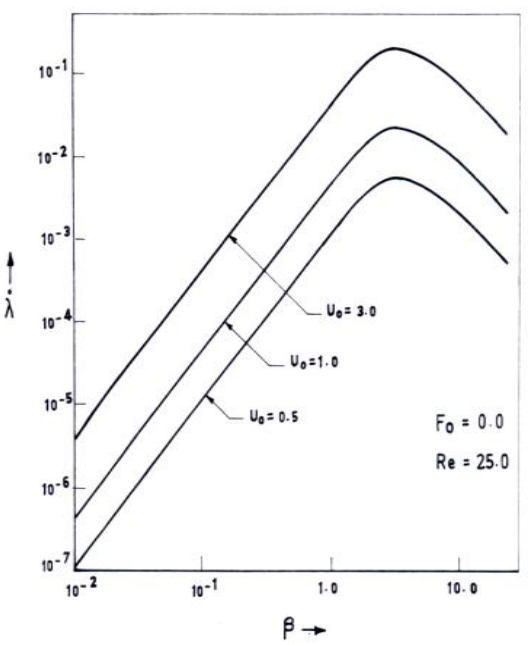

Fig. 3. Effect of oscillation of plates on $\dot{\lambda}$ in the absence of oscillatory body force

Figure 5 portrays the combined effect of $U_{O}$ and $F_{O}$ on $\dot{\lambda}$. The higher the value of these two parameters, in general, the larger is the value of $\dot{\lambda}$. For example when, $U_{O}=3.0$ and $F_{O}=3.0$ approximately $\dot{\lambda}=0.054490$ and the corresponding value of $\kappa_{e}$ is of $\mathrm{O}(1)$, which is several orders of magnitude larger than the original thermal diffusivity $\kappa$ of the fluid. Though one might wish to increase the value of $U_{O}$ and $F_{O}$ indefinitely to achieve better enhancement of heat transfer rates, the limiting factor is that one should ensure laminar flow conditions to avoid the undesirable net mass transfer. The resulting axial heat transport is several orders of magnitude greater than the best possible values achievable with liquid metal heat pipes (Kurzweg 1985b). These results are all in good agreement with the results reported earlier by Kurzweg and Zhao (1984) and Kurzweg (1985b, 1986 b).

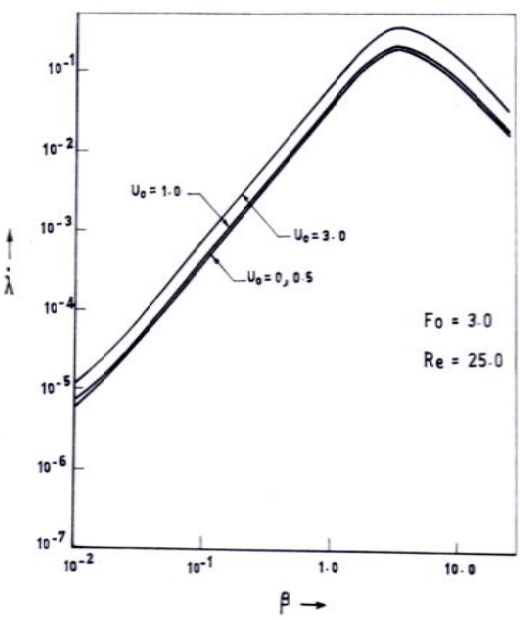

Fig. 4. Effect of oscillation of plates on $\dot{\lambda}$ in the presence of oscillatory body force

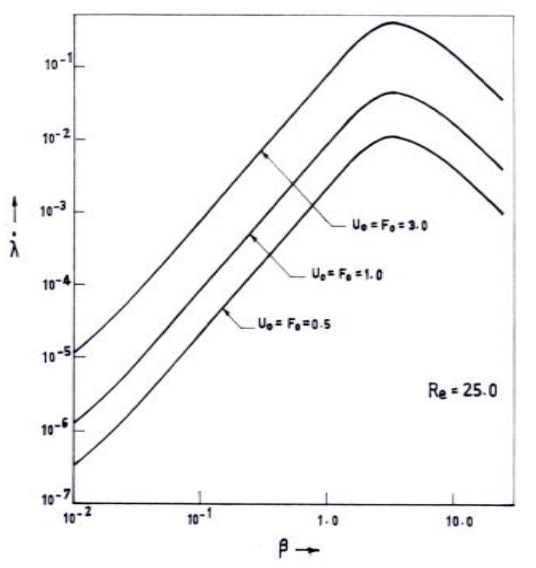

Fig. 5. Combined effect of oscillation of plates and oscillatory body force on $\dot{\lambda}$

\section{Conclusion}

It is inferred that, under constant axial thermal gradient, sinusoidal oscillation of the fluid results in the substantial enhancement of heat transfer and it does not matter how the oscillation was generated. Hence, one might choose the right mode of oscillating the fluid suitable to the desired application. Especially, this result is useful in cooling of electronic devices where one should not disturb the assembly of integrated chips by oscillations.

Finally, it is suggested that the corresponding hydromagnetic problem is worth exploring on account of its wide application in the design of Liquid Metal Magnetohydrodynamic Heat Transfer Systems. 


\section{ACKNOWLEDGEMENTS}

One of the authors (K.S.) wishes to thank Prof. S. Balasundaram for his constant encouragement.

\section{REFERENCES}

Bergles, A.E. (1985). Techniques to augment heat transfer. In Rohsenow, W.M., Hartnett, J.P. and Garnic, E.J. (Eds.), Hand-book of Heat Transfer Applications, $2^{\text {nd }}$ Edition, Mc-Graw Hill (New York), Chapter 3.

Chatwin, P.C. (1975). On the longitudinal dispersion of passive contaminant in oscillatory flows in tubes. Journal of Fluid Mechanics 71(3), 513-527.

Dunn P.D. and D.A. Reay Heat Pipes, $2^{\text {nd }}$ Edition., Pergamon, Oxford.

Grover, G.M., T.P. Cotter, G.F.Erickson (1964). Structures of very high thermal conductance. Journal of Applied Physics 35(6), 1990-1991.

Kaviany, M. (1986). Some aspects of enhanced heat diffusion in fluids by oscillation. International Journal of Heat and Mass Transfer 29(12), 20022006.

Kurzweg, U.H. and L.D. Zhao (1984). Heat transfer by high-frequency oscillations: A new hydrodynamic technique for achieving large effective thermal conductivities. Physics of Fluids 27(11), 26242627.

Kurzweg, U.H. (1985 a). Enhanced Heat Conduction in Fluids Subjected to Sinusoidal Oscillations. Journal of Heat Transfer 107, 459-462.

Kurzweg, U.H. (1985 b). Enhanced heat conduction in oscillating viscous flows within parallel-plate channels. Journal of Fluid Mechanics 156, 291300 .

Kurzweg, U.H. (1986 a). US Patent No.4,590,993 dated May 27, 1986.

Kurzweg, U.H. (1986 b). Temporal and spatial distribution of heat flux in oscillating flow subjected to an axial temperature gradient. International Journal of Heat and Mass Transfer 29(12), 1969-1977.

Manglik, R.K. and A.E. Bergles (1992). Heat transfer enhancement and pressure drop in viscous liquid flows in isothermal tubes with twisted-tape inserts. Warme-und Stoffubertragung 27(4), 249-257.

Merkli, P. and Thomann, H. (1975). Transition to turbulence in oscillating pipe flow. Journal of Fluid Mechanics, 68 (3), 567- 576.
Ozawa, M. and A. Kawamoto (1991). Lumpedparameter modeling of heat transfer enhanced by sinusoidal motion of fluid, Inernational Journal of Heat and Mass Transfer 34(12), 3083-3095.

Shailendhra.K. and S.P. Anjali Devi (1997). Heat transport along an oscillating flat plate in the presence of a transverse magnetic field. International Journal of Heat and Mass Transfer 40(2), 498-501.

Shailendhra.K. (2002). Studies on Unsteady and Enhanced Heat Transfer in MHD Flows. Ph.D. thesis, Bharathiar University, Coimbatore, India.

Shailendhra.K. and S.P. Anjali Devi (2005 February). Temporal and spatial distribution of heat flux in oscillating MHD flow subjected to an axial temperature gradient. In Proceedings of the International Conference on Recent Advances in Mathematics, Gulbarga University, pp.182-193, Gulbarga, India.

Vishwas, V.W. (1998). Improving Industrial Heat Transfer- Compact and Not-So-Compact Heat Exchangers. Journal of Enhanced Heat Transfer 5(1), 53-69.

Zhang, X.R., S. Maruyama, and S. Sakai, (2004). Numerical investigation of laminar natural convection on a heated vertical plate subjected to a periodic oscillation, International Journal of Heat and Mass Transfer 47(19-20), 4439-4448. 